16 | 1994

Varia

\title{
Point de vue et perspective dans les Pensées de Pascal
}

Jean Mesnard

\section{OpenEdition}

\section{Journals}

Édition électronique

URL : http://journals.openedition.org/ccibp/587

DOI : $10.4000 /$ ccibp. 587

ISSN : 2493-7460

Éditeur

Centre international Blaise Pascal

Édition imprimée

Date de publication : 5 avril 1994

Pagination : 3-8

ISSN : 0249-6674

\section{Référence électronique}

Jean Mesnard, «Point de vue et perspective dans les Pensées de Pascal », Courrier du Centre

international Blaise Pascal [En ligne], 16 | 1994, mis en ligne le 07 janvier 2016, consulté le 19 avril 2019. URL : http://journals.openedition.org/ccibp/587 ; DOI : 10.4000/ccibp.587

Ce document a été généré automatiquement le 19 avril 2019

Centre international Blaise Pascal 


\title{
Point de vue et perspective dans les Pensées de Pascal
}

\author{
Jean Mesnard
}

1 L'expression « point de vue » reçoit, dans l'usage courant, un sens très général et abstrait, synonyme d'«opinion particulière». Or son sens premier, dont les bons écrivains tiennent toujours compte, se réfère à une expérience fondamentale. Selon la place qu'occupe l'œil d'un observateur devant un objet, se dessine une image particulière de cet objet. Est-ce donc la place de l'œil qui constitue le point de vue? Avant de répondre, il faut rappeler que l'expression appartient d'abord au vocabulaire technique de la perspective : elle suppose l'existence, non seulement d'un observateur et d'un objet, mais encore celle d'un tableau sur lequel l'objet est représenté. Quelques mots sur cette science sont d'autant plus indispensables qu'elle est en plein essor au début du XVII e siècle, et que Pascal l'a lui-même pratiquée très jeune, parallèlement à ses travaux sur les coniques, domaine très proche, sous la conduite de son premier initiateur à la géométrie, le Lyonnais Girard Desargues. En 1654, quelques mois avant sa grande conversion, il mentionne parmi ses travaux achevés une Méthode de perspective ${ }^{1}$. Un peu plus tôt, dans le très brillant début, seul conservé, de son Traité des coniques, sous le titre Generatio conisectionum $^{2}$, il a recouru abondamment aux techniques de la perspective. Comme plusieurs travaux récents l'ont montré, un détour par la science pascalienne peut constituer la meilleure voie d'accès aux Pensées. Le modèle du genre est fourni par le très brillant chapitre Le Paradigme pascalien, dans l'ouvrage de Michel Serres, Le Système de Leibniz et ses modèles mathématiques ${ }^{3}$, où la perspective est largement prise en compte. Ma recherche pourra recouper la sienne, mais elle se développe dans une orientation différente. Michel Serres se place sur le terrain épistémologique : plutôt que la notion de point de vue, c'est celle de point fixe qui s'impose à son attention, le point fixe, souvent introuvable, par rapport auquel peut seul s'organiser le savoir. Je m'appliquerai surtout, pour ma part, à montrer dans le concept de point de vue, et dans tout ce qui en découle, une technique de réflexion et d'expression dont il faudra traquer la présence et analyser la portée, l'une et l'autre tantôt très visibles, tantôt fort bien dissimulées, dans la série des 
Pensées. On s'apercevra que l'esprit et l'imagination de Pascal s'appliquent tout particulièrement à pratiquer le déplacement du point de vue.

2 Pour effectuer le repérage des textes à lire selon cette grille, je rappellerai sommairement les données fondamentales de la perspective, et surtout son vocabulaire, dont l'emploi éventuel dans les Pensées sera le meilleur indice du recours à ses modèles. Posons d'abord, comme il va de soi, l'œil et l'objet - que Desargues appelle «sujet»: simple question, dirai-je, de " point de vue », ce qui est objet pour l'œil étant sujet pour le tableau. Si l'on veut analyser la vision de l'objet par l'œil, il faut se mettre dans la situation du peintre - du peintre de la Renaissance et de l'âge classique, car chez le peintre primitif, la signification symbolique l'emportant sur la représentation de la réalité, la perspective a peu d'importance et, depuis latin $d u X X^{e}$ siècle, cette discipline a été amplement contestée. Il faut aussi se faire géomètre, construire rigoureusement, à partir de l'objet réel, l'image de l'objet. Ce qui suppose la constitution d'un système de références. Il comprend d'abord le tableau, sur lequel l'objet, situé dans un espace à trois dimensions, doit être représenté en toute exactitude dans un espace à deux dimensions. Plutôt que «tableau », le géomètre dira " plan du tableau ». Celui-ci est naturellement situé entre l'œil et l'objet, et supposé transparent pour les nécessités de l'analyse : FGHI sur la figure donnée par le Dictionnaire mathématique d'Ozanam ${ }^{4}$. Ce plan s'appuie perpendiculairement sur un autre, le "plan géométral », figurant idéalement la terre et parallèle à l'horizon, $A B C D$ sur la figure: c'est sur lui que sont représentés, par exemple, les plans d'architectes.

Perspective constituée à partir d'un " plan de tableau »

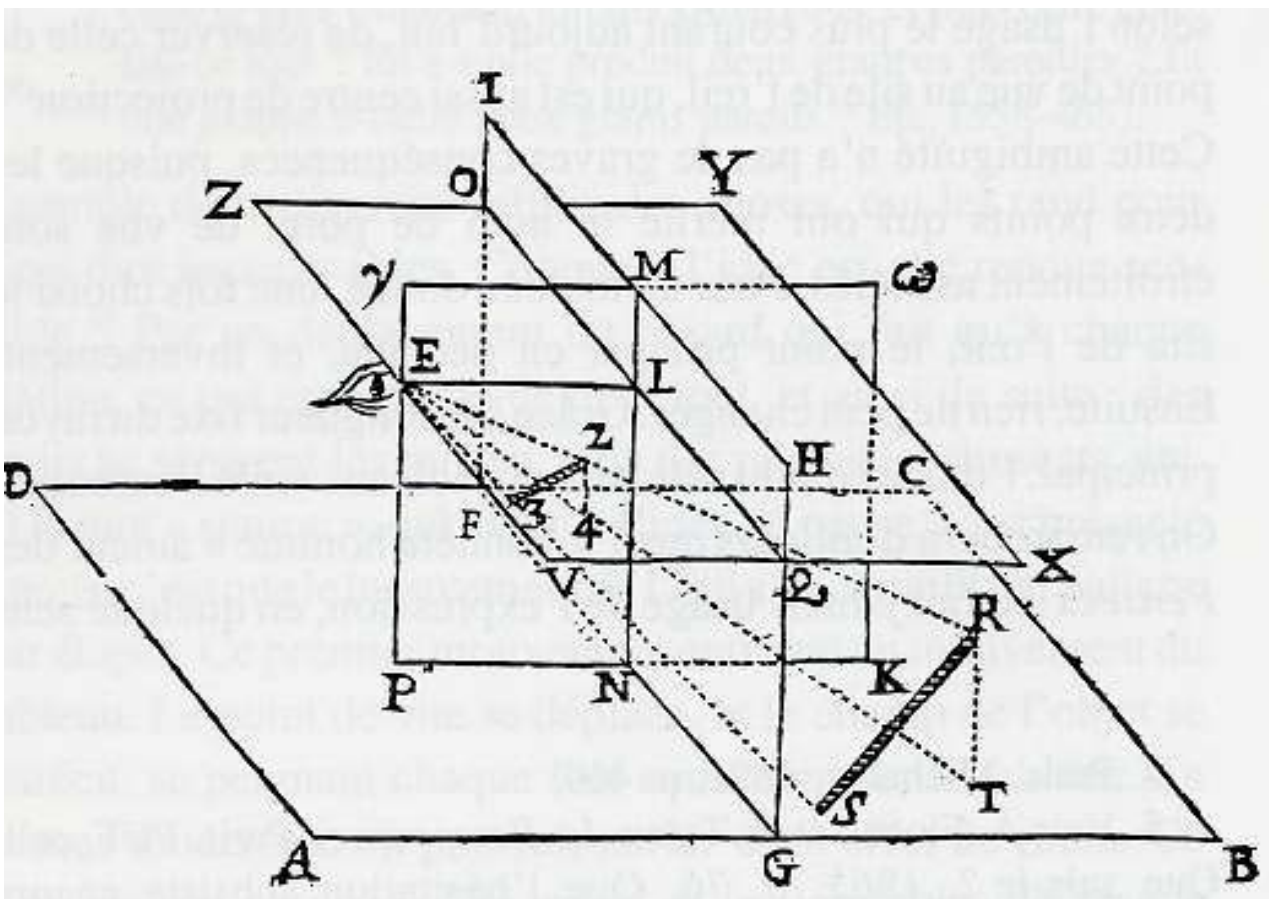

Dictionnaire mathématique d'Ozanam

Mais deux plans ne suffisent pas, dans l'espace, pour obtenir les coordonnées d'un point, notamment, ici, celles de l'œil ; il en faut trois. D'autres plans vont précisément passer par l'œil : le plan vertical, perpendiculaire, d'une part au plan géométral, d'autre part au plan 
du tableau : ce sera PK, oméga, gamma; le plan horizontal, parallèle au plan géométral et perpendiculaire au plan du tableau: VXYZ. L'intersection de ces deux derniers plans s'effectue selon une ligne, EL sur la figure, que l'on appelle « rayon principal ». C'est le point $\mathrm{L}$, projection de l'œil sur le tableau, qui, dans la langue de Desargues et dans celle du XVII siècle en général, et souvent encore aujourd'hui, est le point de vue, appelé aussi « point de l'œil » ou « point principal ». C'est autour de lui que s'organise la composition: il appartient à la ligne verticale médiane du tableau, et à la ligne horizontale par rapport à laquelle se distribuent les points de l'objet, les uns au-dessus, les autres au-dessous selon qu'ils se situent au-dessus ou au-dessous du plan horizontal. Appelé plutôt aujourd'hui «point de fuite» ou "point de fuite principal », il est celui vers lequel se dirigent, sur le tableau, les lignes qui, dans l'objet, sont perpendiculaires au plan du tableau: songeons à quelques représentations célèbres de carrelages en damier. Cette notion de point de fuite a permis, selon l'usage le plus courant aujourd'hui, de réserver celle de point de vue au site de l'œil, qui est aussi centre de projection ${ }^{5}$. Cette ambiguïté n'a pas de graves conséquences, puisque les deux points qui ont mérité le nom de point de vue sont étroitement associés. Pour un tableau donné, une fois choisi le site de l'œil, le point principal en découle, et inversement. Ensuite, rien ne peut changer. Grâce à la longueur fixe du rayon principal, l'œil est rendu solidaire du tableau : voilà l'essentiel. On remarquera d'ailleurs que l'« honnête homme » auteur des Pensées ne fait jamais usage de l'expression, en quelque sens que ce soit. Mais il n'oublie pas pour autant le schéma perspectif fondamental.

4 De cet ensemble de données, ce qu'il retient de façon explicite - mais il ne faut pas négliger l'implicite -, c'est essentiellement ce qui entre dans le langage commun et peut être accepté par l'esprit de tous : le regard de l'œil, l'objet donné et la trace de cet objet sur le tableau, déterminée par les lois de la perspective. En ce dernier domaine, le vocabulaire de Desargues, tel qu'il le propose dans sa Méthode universelle de perspective publiée en 1636, offre quelques termes qu'il importe de retenir. Entamant son ouvrage, le géomètre dit notamment :

Les mots perspective, apparence, représentation et pourtrait y sont chacun le nom d'une même chose.

Conséquence :

Les mots représenter, pourtraire, trouver l'apparence, faire ou mettre en perspective y sont employés en même signification l'un que l'autre.

Plus loin :

«Ce que d'aucuns nomment plan géométral, autres plan de terre, autres la plante du sujet, y a nom assiette du sujet ", ou, plus rigoureusement, plan de l'assiette du sujet. Chaque point $d u$ sujet a naturellement une assiette particulière, la projection orthogonale de ce point sur le plan géométral ${ }^{6}$.

5 Moins typiquement " arguésiens », pour employer l'adjectif que les spécialistes ont forgé d'après « cartésien ", d'autres termes employés dans les Pensées appartiennent aussi au langage de la perspective. Il m'appartiendra, le cas échéant, de montrer la portée de leur présence.

6 Voilà terminée la partie préparatoire - la plus difficile - de notre enquête. Entrer dans le corps du sujet n'est rien d'autre que de montrer toutes les applications possibles - et nécessaires - de ces données théoriques et techniques pour l'interprétation des Pensées. Tâche simplifiée par le fait que Pascal n'envisage que des situations très simples et fait jouer essentiellement - quoiqu'il évite l'expression - la notion de point de vue. 
7 L'ordre à suivre sera celui du concret à l'abstrait ou, plus précisément, des allusions directes aux modèles de la perspective, à ce qui, dans ces modèles, informe les structures de la pensée.

8 L'exemple le plus concret est fourni par un texte bien connu, concernant la difficulté de trouver le juste lieu pour bien juger :

[...] Si on considère son ouvrage incontinent après l'avoir fait, on en est encore tout prévenu, si trop longtemps après, on n'y entre plus.

Ainsi les tableaux vus de trop loin et de trop près. Et il n'y a qu'un point indivisible qui soit le véritable lieu. Les autres sont trop près, trop loin, trop haut ou trop bas. La perspective l'assigne dans l'art de la peinture [...] (21-55)7.

Le commentaire est aisé : il faut que l'œil - un œil unique, postulat de la perspective classique - se place au point idéal où l'artiste le plaçait, et qu'il a choisi pour organiser sa composition; point assigné par la perspective et commandé par la localisation du point principal sur le tableau. Dans le domaine tout humain de l'art, il est donc possible de trouver la référence à partir de laquelle le jugement pourra s'exercer correctement. Les sens viennent au secours de ce dernier.

Ce n'est là qu'un exemple illustrant une pensée en elle-même abstraite ; et où la solution trouvée pour la peinture ne se retrouvera pas. Dans cette sorte d'entre-deux où nous ne cessons de vivre, il est pratiquement impossible de trouver le bon lieu pour juger :

Si on est trop jeune, on ne juge pas bien, trop vieil de même. Si on n'y songe pas assez, si on y songe trop, on s'entête et on s'en coiffe [...] (21-55).

Trop et trop peu de vin. Ne lui en donnez pas, il ne peut trouver la vérité. Donnez-lui en trop, de même (38-72).

Quand on lit trop vite ou trop doucement, on n'entend rien (41-75 cf. 723-601).

Quand le jugement n'a pas de référence solide, il ne peut trouver l'image exacte des choses. C'est le cas « dans la vérité et dans la morale » (21-55).

Cette impossibilité de trouver le bon lieu entraîne l'« inconstance » humaine :

Je n'ai jamais jugé d'une même chose exactement de même. Je ne puis juger d'un ouvrage en le faisant : il faut que je fasse comme les peintres et que je m'en éloigne, mais non pas trop. De combien donc? Devinez (558-465 fin).

Deviner, en l'occurrence, est chose impossible. Les peintres n'ont qu'à retrouver le point de vue. Dans les jugements humains, ce point est introuvable.

On peut, dans un texte de signification voisine, reconnaître les situations caractéristiques de la perspective :

Les choses ont diverses qualités et l'âme diverses inclinations, car rien n'est simple de ce qui s'offre à l'âme, et l'âme ne s'offre jamais simple à aucun sujet. De là vient qu'on pleure et qu'on rit d'une même chose (54-87).

14 L'âme est comme l'œil de l'esprit ; « sujet » est le mot technique employé par Desargues pour désigner ce qui sera représenté sur le tableau. Les variations de l'âme et les éléments variés du sujet, appelant le choix de plusieurs points de vue, introduisent diversité dans l'aspect du tableau.

15 C'est encore le vocabulaire qui nous invite à nous arrêter sur d'autres fragments. On se souvient que, pour Desargues, "portrait» est synonyme de représentation sur un tableau; si bien que le mot n'a pas le sens que nous lui donnons aujourd'hui lorsque nous distinguons, par exemple, un portrait d'un paysage. D'où le commentaire à donner au fragment bien connu :

L'éloquence est une peinture de la pensée. Et ainsi, ceux qui, après avoir peint, ajoutent encore, font un tableau au lieu d'un portrait (578-481). 
La pensée est l'objet (ou le sujet). L'éloquence est la peinture. Cette peinture est portrait si elle représente purement et simplement l'objet, interprété seulement selon les lois de la perspective et le choix du point de vue. Si la fantaisie s'y introduit, la fidélité à l'objet disparaît ; les lois du naturel sont violées. En extrapolant au-delà de la peinture, lorsque Pascal considère les figures de l'Ancien Testament, on voit revenir le terme " arguésien » :

Un portrait porte absence et présence, plaisir et déplaisir, La réalité exclut absence et déplaisir (260-291; cf. 265-296, où figure remplace significativement portrait).

17 L'argumentation vise à montrer que les prophètes, parlant de la loi et des sacrifices, y voyaient « autre chose » dont leurs propos étaient « la peinture ». «Car dans un portrait on voit la chose figurée.» Toutes les considérations sur les figures se trouvent donc susceptibles d'une interprétation de type perspectif. Le point de vue des juifs n'est pas celui des chrétiens ou, plus exactement, les juifs, ne prenant aucun recul vers le juste point de vue, confondent la peinture et l'objet ; seuls les chrétiens savent les distinguer.

18 Jusqu'ici, le modèle perspectif a toujours été utilisé de manières comparables; en particulier, le tableau est toujours demeuré immobile. Mais la validité du modèle reste entière dans des cas plus complexes, où sa présence est plus difficile à saisir, quoique toute abstraction soit exclue. La pensée n'est pas des plus connues, mais elle est des plus fascinantes :

On distingue des fruits les raisins, et entre ceux-là les muscats, et puis Condrieu, et puis Desargues, et plus cette ente. Est-ce tout? En a-t-elle produit deux grappes pareilles? Et une grappe a-t-elle deux grains pareils ? Etc. (558-465).

19 Exemple de la diversité infinie des choses, qui les rend pour ainsi dire insaisissables. Comment l'idée est-elle rendue sensible? Par un déplacement du regard qui fait qu'à chaque station, ce qui était partie devient tout, et ainsi de suite : des fruits se séparent les raisins ; puis des raisins les muscats, etc. Si le mot «station ", qui évoque l'idée de pause, s'est présenté à nous, c'est que le mouvement de l'œil s'accomplit par paliers, par étapes. Ce premier mouvement entraine un mouvement du tableau. Le point de vue se déplace, et le champ de l'objet se rétrécit, se peignant chaque fois autrement sur le tableau. En termes modernes, on pourrait parler d'un effet de zoom. Ce n'est pas un hasard si Desargues et les vignobles de grande réputation qu'il possédait à Condrieu, près de Lyon, sont présents dans cette succession d'images. Car ce texte est doublement " arguésien » : le personnage de Desargues en est le centre; et la perspective, dont ce géomètre a été le maitre, y est maniée avec virtuosité. Jusqu'ici ce fragment a surtout retenu l'attention parce qu'il suggérait l'idée d'une visite de Pascal à Condrieu, chez Desargues. Hypothèse fort plausible. Mais il en est une autre, qui n'a pas encore été formulée. Ce qui fournit à Pascal l'occasion d'une série de tableaux successifs n'était-il pas déjà un tableau, décorant l'intérieur de Desargues à Paris, où il séjourna longtemps? N'oublions pas, en dernier lieu, que l'imagination a pu, en fait, tenir la place de la vue ? En tout cas, il y a beaucoup mieux à dire sur ce fragment que ce qu'il peut enfermer d'anecdote.

Il subsiste dans ce fragment une imprécision, sans doute parfaitement consciente. Dans son mouvement, dans ses stations successives, le regard va-t-il du premier plan à l'arrière-plan ou inversement? En d'autres termes, la diversité des fruits, puis des raisins est-elle ce qui parait au plus près, le regard se concentrant ensuite en progressant ? ou bien le spectacle d'ensemble se situe-t-il à l'horizon, les détails se faisant de plus en plus précis au fur et à mesure que le regard se rapproche? La difficulté de répondre à cette double question est d'autant plus significative qu'elle n'existe pas dans les fragments comparables auxquels il convient aussi de s'arrêter: le sens du mouvement y est 
parfaitement indiqué. C'est la comparaison avec ces derniers qui imposera l'idée d'un mouvement d'avant en arrière : un tel mouvement est en effet de règle en cas de passage du grand au petit ${ }^{8}$.

21 Appliquons-nous en effet à un autre fragment, non moins décourageant pour la critique, et relatif aussi à l'idée de diversité :

Une ville, une campagne, de loin c'est une ville et une campagne; mais à mesure qu'on s'approche, ce sont des maisons, des arbres, des tuiles, des feuilles, des herbes, des fourmis, des jambes de fourmis, à l'infini. Tout cela s'enveloppe sous le nom de campagne (65-99).

Il s'agit toujours de passer du tout aux parties, par une série de subdivisions. Mais, si le mouvement va bien de l'arrière vers l'avant, cette précision n'est pas la seule différence avec le fragment précédent. On voit d'abord deux objets se subdiviser parallèlement : une ville, une campagne, l'ensemble constituant les deux « apparences » du monde terrestre : par quoi le parallélisme met lui-même en valeur la diversité. C'est la campagne qui est analysée le plus longuement : aussi bien occupe-t-elle la plus grande partie de l'espace, et un espace dans lequel la ville elle-même se situe. Plus important: la subdivision ne s'opère pas de la même façon que pour les raisins de Condrieu : elle ne porte plus sur des collections d'objets qui, se faisant plus menus, deviennent de plus en plus semblables mais sur un objet unique, que l'on voit peu à peu se décomposer en éléments, cette décomposition pouvant se poursuivre "à l'infini ". À quoi il faudrait ajouter: sans que l'on n'arrive jamais au néant. L'effet de zoom précédent se renouvelle; mais les lois de la perspective, ce qui n'a rien de surprenant, appellent une mise en relation avec la théorie des indivisibles. Il faut cependant écarter toute identification des infiniment petits auxquels conduit la décomposition des objets successifs avec des indivisibles. Car les indivisibles n'ont pas de parties. Les infiniment petits saisis au terme du regard perspectif ont encore une étendue. Ils sont de même genre que l'étendue ; ils appartiennent au même ordre. C'est ce que Pascal déclare très fermement à la fin du premier fragment de L'Esprit géométrique, où il utilise, avec d'autres, le même exemple que dans les Pensées. Une ville et une maison sont des grandeurs de même genre. Certes "mille maisons font une ville, quoique aucune ne soit ville ». Mais c'est là une simple question de langage : «On a donné le nom [...] de ville à plusieurs maisons. » Mais d'une maison à une ville, il n'y a différence que de quantité. D'ailleurs, "quoiqu'une maison ne soit pas une ville, elle n'est pas néanmoins un néant de ville ${ }^{9}$ ». Les points de vue successifs n'ont pas de fin.

D'une manière concrète, il est clair, à suivre ce fragment, que l'œil et le tableau, qu'ils progressent vers la ville ou vers la campagne, font une série de stations devant des objets de plus en plus petits, le mouvement s'arrêtant aux limites du visible, non sans que s'offre la possibilité d'aller au-delà.

24 Le modèle perspectif peut aussi se découvrir dans des développements beaucoup plus abstraits. À cet égard, une attention spéciale doit être apportée à un fragment célèbre de la liasse Raison des effets :

Gradation. Le peuple honore les personnes de grande naissance. Les demi-habiles les méprisent, disant que la naissance n'est pas un avantage de la personne, mais du hasard. Les habiles les honorent, non par la pensée du peuple, mais par la pensée de derrière. Les dévots, qui ont plus de zèle que de science, les méprisent, malgré cette considération qui les fait honorer par les habiles, parce qu'ils en jugent par une nouvelle lumière que la piété leur donne. Mais les chrétiens parfaits les honorent par une autre lumière supérieure. 
Ainsi se vont les opinions succédant du pour au contre, selon qu'on a de lumière (90-124). exégèse particulièrement brillante ${ }^{10}$. Il en a vu le modèle dans la théorie perspective des coniques, poussée et systématisée par Pascal à partir des découvertes de Desargues. Il s'est appuyé en premier lieu sur le seul morceau qui nous ait été conservé, grâce à une copie due à Leibniz, d'un Traité des coniques demeuré inachevé, morceau déjà signalé, intitulé Generatio conisectionum. Toutes les coniques sont engendrées par l'intersection du cône avec un plan. Dans le cas le plus simple, où le plan est perpendiculaire à l'axe, la conique est un cercle. L'œil se situant au sommet, toutes les autres coniques sont l'image du cercle, considéré comme l'objet, sur un autre plan sécant : ainsi se dessinent l'ellipse, la parabole et l'hyperbole. Or cercle et ellipse sont fermes et finis, parabole et hyperboles sont infinies : l'ouverture de ces dernières s'explique, comme le montre Pascal, par le fait d'un ou deux " points manquants ». Le renversement du pour au contre serait représenté par cette succession de courbes fermées et ouvertes. Un ordre s'instaure pour l'œil établi au point de vue.

La richesse des analyses de Michel Serres ressort mal d'une telle simplification. Mais il est d'autant plus naturel de contester la pertinence du modèle proposé que l'auteur en a lui-même perçu les insuffisances. Il reconnaît la difficulté de reconnaître une véritable progression, une véritable « gradation » dans le passage du cercle à la parabole, à l'ellipse et à l'hyperbole, prolongé par un retour final au cercle. Aussi suggère-t-il de compléter le modèle conique par d'autres empruntés aux écrits sur la roulette, de même qu'il avait commencé par invoquer celui des carrés magiques ${ }^{11}$. C'en est peut-être trop : l'excès de modèles ne dénonce-t-il pas l'absence de modèle?

Dans le détail, deux expressions remarquables de Pascal demeurent aussi sans explications. Pourquoi le « renversement du pour au contre » comporte-t-il l'acquisition d'une «lumière » croissante? Ce n'est pas répondre à cette question que d'appeler la géométrie de Desargues, une "géométrie de l'ombre et de la lumière ${ }^{12}$ ». Quant à l'expression " pensée de derrière ", elle est complètement négligée.

Michel Serres oriente pourtant heureusement la réflexion, sans aller jusqu'au bout, en montrant la parenté qui unit la "gradation » de la « raison des effets » avec la phrase finale d'un fragment sur le « roseau pensant » :

Par l'espace, l'univers me comprend et m'engloutit comme un point ; par la pensée, je le comprends (113-145). À quel modèle perspectif pourrait-on rattacher ces images grandioses?

Nous avons pu observer précédemment l'œil et le tableau se déplaçant vers l'avant pour aller du plus grand au plus petit... Sur ce nouvel exemple, c'est un effet d'agrandissement qui se produit. N'est-ce pas à dire que l'œil et le tableau se déplacent vers l'arrière pour que la vue s'étende, par stations successives, du plus petit au plus grand? Quoiqu'un langage plus abstrait dissimule d'abord la réalité de ce mouvement, on ne peut, me semble-t-il, s'y tromper.

31 Selon un premier point de vue, l'œil et le tableau ne visent qu'à embrasser un objet proche et limité : le «je » qui parle, c'est-à-dire l'homme, conçu comme corps. Si la vue s'éloigne, ce «je » se réduit à un point et, le champ de vision s'agrandissant, apparaît l'univers, sphère en tant qu'objet, cercle sur le tableau, sorte de contraire du point qu'il « comprend ». Que la vue s'éloigne encore, c'est l'univers à son tour qui se réduit à un 
point, absorbé qu'il est par le «je » conçu comme pensée, réalité abstraite, mais que la reprise de "comprend» invite à saisir de nouveau comme sphère et comme cercle opposés à un point. Voilà encore un «renversement du pour au contre ", qui ramène à l'objet initial, pourvu toutefois d'un sens plus riche par le chemin parcouru, par l'inclusion des étapes initiales dans l'étape finale. Un progrès s'est bien accompli vers la "lumière ", au sens abstrait de connaissance, mais aussi en un sens concret : il faut plus de lumière pour voir loin que pour voir près. Quant à l'expression " pensée de derrière ", elle convient à merveille à ce mouvement de l'avant vers l'arrière qui fait passer d'un savoir partiel à un savoir total par inclusion progressive des parties dans un tout.

Selon cette analyse, le point garde toujours une certaine consistance et les cercles grandissants ne sont pas tenus pour infinis. Nous demeurons dans le réalisme de la peinture, sans entrer dans l'absolu de la géométrie. On observera cependant que le mouvement conduit à passer du premier au second des fameux «ordres» de grandeurs, qui, selon le fragment bien connu (308-339) sont infiniment distants.

Appliquons ce modèle à la " gradation " précédemment citée. Dans une première position l'œil et le tableau embrassent l'objet "peuple", avec ses opinions. Lorsque la vue s'éloigne, l'opinion du peuple se réduit à un point et, le champ s'agrandissant, apparait, comme un cercle, celle des "demi-habiles", qui en est le contraire. Le mouvement se poursuivant, c'est l'opinion des demi-habiles qui devient point et celles des habiles cercle: les deux opinions précédentes, qu'elle comprend, y subsistent l'une et l'autre, mais chacune avec sa vérité particulière, l'opinion du peuple vraie pour le comportement et fausse pour la pensée, l'opinion des demi-habiles vraie pour la pensée et fausse pour le comportement (cf. 92-93, 126-127). L'opinion des habiles inclut les deux points représentatifs des opinions précédentes; mais s'en tenir à l'un ou l'autre de ces deux points est tomber dans l'erreur.

Le mouvement se poursuit de la même manière avec les dévots et les chrétiens parfaits. Le terme auquel nous parvenons alors est-il définitif? C'est ce que pense Michel Serres. En fait, si le mouvement peut s'arrêter chaque fois que le point de départ est retrouvé, il peut aussi se prolonger à l'infini, comme le montre au moins un fragment susceptible d'être analysé selon le même modèle (122-155).

Pour revenir au concret, il est indispensable de s'arrêter maintenant au grand fragment Disproportion de l'homme (199-230). On y progresse, d'une part, vers l'infiniment grand, d'autre part, vers l'infiniment petit. C'est l'occasion de confirmer les analyses précédentes. Les deux modèles, ou plutôt les deux variantes du modèle constitué par le déplacement de l'œil et du tableau, soit vers l'avant, soit vers l'arrière, s'y conjuguent de la façon la plus brillante. Le mouvement vers l'arrière est celui qui convient pour aller par étapes vers l'infiniment grand. L'observateur situé sur la terre, prenant du recul, voit celle-ci se réduire à un point devant la révolution du soleil. Adoptant encore un site plus éloigné, il voit cette révolution se réduire à son tour à un point devant celle des astres. Puis l'imagination - autre expression de la vue - prend le relais, et se place à son tour en des sites de plus en plus éloignés, avec des champs de plus en plus vastes, jusqu'à l'infini. On découvre ainsi que, si le regard, extérieur ou intérieur, mène de plus en plus loin, l'œil, toujours accompagné du tableau, recule aussi, en sens inverse, à l'infini. Un infini de petitesse se constitue donc implicitement lorsque la vue s'efforce d'atteindre l'infiniment grand : les extrêmes sont inséparables. de l'infiniment petit. Mais la différence était déjà inscrite dans les deux variantes de notre 
modèle. Le mouvement vers l'infiniment petit s'accomplit par déplacement de l'œil et du tableau vers l'avant. Mais les objets successifs qui se présentent sont comparables à ceux que rencontre l'observateur qui découvre progressivement la ville et la campagne, passant du tout aux parties les plus ténues. Le ciron que dissèque l'auteur de Disproportion de l'homme rappelle les fourmis, puis les jambes de fourmis rencontrées par le voyageur inventoriant les réalités de la campagne. Mais ce dernier s'arrête aux limites du visible. Dans Disproportion de l'homme, nous allons jusqu'à l'invisible, dont l'esprit ne peut rendre compte que par des images empruntées à l'infiniment grand. Une fois de plus, les extrêmes se rejoignent.

Que de tels modèles soient consciemment utilisés au long de ce fragment, on en trouvera confirmation dans le grand nombre des termes empruntés à la perspective que l'on peut y relever. L'homme ballotté entre les deux infinis recherche une " assiette ferme ». C'est par référence à la perspective qu'on peut parler d'un « raccourci d'atome ». Enfin si l'homme, dans sa "disproportion", ne peut rien faire «sinon d'apercevoir quelque apparence du milieu des choses », le mot "apparence» est évidemment à prendre au sens que lui accorde Desargues, celui de représentation sur un tableau. Non seulement le savoir ne porte que sur le « milieu des choses ", qui seul a proportion avec l'homme, mais il met en présence, non de l'objet, mais de son image. Il est soumis aux lois de la vision, que l'on pourrait identifier avec celles du langage.

Il y aurait lieu de chercher dans les Pensées d'autres applications des modèles déjà définis et surtout la présence d'autres modèles qui puisent leur origine dans la perspective. Entre ces derniers nous n'en retiendrons qu'un, d'une importance exceptionnelle :

Quand on veut reprendre avec utilité et montrer à un autre qu'il se trompe, il faut observer par quel côté il envisage la chose, car elle est vraie ordinairement de ce côté-là, et lui avouer cette vérité, mais lui découvrir le côté par où elle est fausse. Il se contente de cela, car il voit qu'il ne se trompait pas et qu'il manquait seulement à voir tous les côtés. Or on ne se fâche pas de ne pas tout voir, mais on ne veut pas être trompé. Et peut-être que cela vient de ce que naturellement l'homme ne peut tout voir, et que naturellement il ne se peut tromper dans le côté qu'il envisage, comme les appréhensions des sens sont toujours vraies (701-579).

Négligeons, dans le commentaire, la référence implicite à l'idéal de l'« honnête homme ", qui, dans son souci de plaire, se garde de contredire autrui ; et, parallèlement, ce qui touche à la rhétorique de la conversation, très différente de la logique, qui fournit les lois du raisonnement. On observera principalement que tout objet - terme qui, en vertu de la métaphore usuelle qui fait désigner par les opérations de la vue celles de l'esprit, désigne ici une idée, ou un ensemble d'idées - peut être envisagé selon une multiplicité de points de vue. Points de vue qui peuvent, soit se situer sur le même axe, comme dans la plupart des exemples précédents, soit sur des axes différents, permettant de voir l'objet sous ses différentes faces. La vérité est la somme des regards portés à partir de ces différents points de vue, ou, si l'on suit le langage courant, la somme de ces points de vue. Les hommes, en général, se contentent d'un seul point de vue, ou de points de vue partiels. Ils se trouvent donc opposés les uns aux autres, tous par défaut de lumière. L'union se rétablira, dans la vérité, lorsque tous les points de vue seront adoptés simultanément.

Il n'est pas impossible d'établir une relation entre cette conception de la vérité et celle, non moins pascalienne, qui la fait dériver de l'union des contraires. En effet, s'il y a contradiction entre les différents points de vue susceptibles d'être pris sur un objet, il est possible de synthétiser leur somme en deux points de vue principaux et contraires. On 
voit combien d'affinités peuvent se découvrir, à la faveur de la perspective, entre des aspects fort divers des Pensées.

Le modèle perspectif, constitué par un ensemble qui comprend l'œil, l'objet et, entre eux, le tableau, et où un rôle capital est joué par le point de vue, aux deux sens que peut, techniquement, recevoir le mot, ou, plus précisément, par le rapport établi, selon le choix de l'artiste, entre l'œil et le « point principal » du tableau, est donc présent au cœur de la pensée de Pascal. La généralité de son emploi tient à la relation métaphorique unissant la vue et la pensée. Elle tient aussi à ce que Pascal voit autant qu'il raisonne, qu'il montre autant qu'il démontre. Sa pensée s'appuie sur un fondement géométrique, mais non pas de n'importe quelle géométrie. La sienne est celle de Desargues, antithèse de celle de Descartes en ce qu'elle est concrète et non abstraite, qu'elle est à la fois rigoureuse et paradoxale - établissant par exemple une identité de structure entre droites parallèles et droites concourantes -, qu'elle se relie étroitement à des disciplines pratiques dont la perspective est sans doute la principale. Une telle géométrie demande beaucoup à l'imagination, cette faculté dont Pascal a dit beaucoup de mal, mais dont il sait qu'il existe un bon usage et que, correctement maniée, elle conduit, non pas à l'erreur, mais à l'illumination.

\section{NOTES}

1. Voir mon édition de Pascal, Euvres complètes, t. II, Paris, Desclée De Brouwer. Bibliothèque européenne, 1970, p. 1034.

2. Ibid., p. 1108-1119.

3. T. II, Paris, P.U.F., 1968, p. 647-712.

4. Paris, Michallet, 1691, p. 469.

5. Voir A. Flocon et R. Taton, La Perspective, Paris, P.U.F., coll. Que sais-je ?, 1963, p. 76. Que l'hésitation subsiste encore aujourd'hui sur ce qu'il faut appeler "point de vue ", on en a la preuve saisissante dans deux écrits successifs d'un même auteur, d'ailleurs éminent, Marisa Dalai Emiliani, qui, dans sa préface, datée de 1961, à la traduction de l'ouvrage capital d'Erwin Panofsky, La perspective comme forme symbolique, Paris, Les Éditions de Minuit, 1975, p. 12-13, identifie, comme le XVII ${ }^{\mathrm{e}}$ siècle, point de vue et point principal, appelant le lieu occupé par l'œil centre de vision, et qui, dans son précieux article Perspective de l'Encyclopodia Universalis, t. XII, 1972, p. 833, ramène le point de vue à ce centre de vision.

6. Desargues, CEuvres, éd. Poudra, t. I, Paris, Leiber, 1864, p. 55-57.

7. Dans nos références aux Pensées, le premier chiffre renvoie à l'édition Lafuma, dans Pascal, Euvres complètes, Paris, Éd. du Seuil, coll. l'Intégrale, 1963 ; le second à l'édition des Pensées par Philippe Sellier, Paris, Bordas, Class. Garnier, 1991, rééd. 1993.

8. Cette imprécision pourrait expliquer le fait que, dans le classement que Pascal a effectué de ses notes, ce fragment, au lieu de paraître aux côtés de ceux que nous allons examiner, soit rangé tout à fait à part, dans une liasse intitulée Miscellanea.

9. Pascal. Euvres complètes, éd. citée, t. III, 1991, p. 407-408.

10. Op. cit., t. II, p. 687-698.

11. Ibid., p. 593-596. 
12. Ibid., p. 592.

INDEX

Mots-clés : Pascal, Pensées, point de vue, perspective

Keywords : point of view

\section{AUTEUR}

\section{JEAN MESNARD}

Professeur émérite de littérature française

Université de Paris IV 